\title{
On the Number of Positive Solutions of a Quasilinear Elliptic Problem
}

\author{
Giovany M. Figueiredo \& Marcelo F. Furtado
}

ABSTRACT. We obtain multiplicity of positive solutions for the quasilinear equation

$$
\begin{aligned}
-\varepsilon^{p} \operatorname{div}\left(a(x)|\nabla u|^{p-2} \nabla u\right)+u^{p-1}= & f(u) \text { in } \mathbb{R}^{N}, \\
& u \in W^{1, p}\left(\mathbb{R}^{N}\right),
\end{aligned}
$$

where $\varepsilon>0$ is a small parameter, $1<p<N, f$ is a subcritical nonlinearity and $a$ is a positive potential such that $\inf _{\partial \Lambda} a>$ $\inf _{\Lambda} a$ for some open bounded subset $\Lambda \subset \mathbb{R}^{N}$. We relate the number of positive solutions with the topology of the set where $a$ attains its minimum in $\Lambda$. The result is proved by using LjusternikSchnirelmann theory.

\section{INTRODUCTION}

Several physical phenomena related to equilibrium of continuous media are modeled by the problem

$$
-\operatorname{div}(a(x) \nabla u)=g(x, u) \text { in } \Omega, \quad u=0 \text { on } \partial \Omega,
$$

where $\Omega$ is a domain of $\mathbb{R}^{N}, g$ is a regular function and $a$ is a nonnegative weight (see [15]). There is an extensive literature about the regularity and spectral theory of the above problem when $g(x, u) \equiv g(u)$ is a linear function (see $[3,5,8,23]$ and references there in). Concerning the nonlinear problem we can cite $[9,10$, 24-26].

In [11], Chabrowski studied the problem

$$
-\operatorname{div}(a(x) \nabla u)+\lambda u=K(x)|u|^{q-2} u \quad \text { in } \mathbb{R}^{N},
$$


with $\lambda>0,2<q<2 N /(N-2)$, and $a \in C\left(\mathbb{R}^{N}\right) \cap L^{\infty}\left(\mathbb{R}^{N}\right)$ satisfying

$$
0 \leq a(x) \leq \lim _{|x| \rightarrow \infty} a(x)
$$

and being positive in the exterior of some ball $B_{R}(0)$. By using minimization arguments he obtained a nonzero solution of (1.1) belonging in some appropriated subspace of $W^{1,2}\left(\mathbb{R}^{N}\right)$. In his result, it was also supposed an integrability condition for $a(x)$ and that $K \in L^{\infty}\left(\mathbb{R}^{N}\right)$ verified either $K(x) \geq \lim _{|x| \rightarrow \infty} K(x)$ or $K$ is periodic.

More recently, Lazzo [19] considered the equation (1.1) with $K \equiv 1$ and the function $a$ satisfying

$$
0<a_{0}:=\inf _{x \in \mathbb{R}^{N}} a(x)<a_{\infty}:=\liminf _{|x| \rightarrow \infty} a(x) .
$$

She proved that, for $\lambda$ sufficiently large, there is an effect of the topology of the set $\left\{x \in \mathbb{R}^{N}: a(x)=a_{0}\right\}$ on the number of positive solutions of (1.1).

Motivated by [19], we are interested in studying the number of positive solutions of a nonhomogeneous quasilinear form of equation (1.1) under a local condition on the potential $a$. More precisely, we deal with the problem

$$
\begin{cases}-\varepsilon^{p} \operatorname{div}\left(a(x)|\nabla u|^{p-2} \nabla u\right)+u^{p-1}=f(u) & \text { in } \mathbb{R}^{N}, \\ u \in C_{\operatorname{loc}}^{1, \alpha}\left(\mathbb{R}^{N}\right) \cap W^{1, p}\left(\mathbb{R}^{N}\right), u>0 & \text { in } \mathbb{R}^{N},\end{cases}
$$

where $\varepsilon>0$ is a small real parameter, $1<p<N, 0<\alpha<1$, and the potential $a: \mathbb{R}^{N} \rightarrow \mathbb{R}$ is continuous and verifies

$\left(a_{1}\right) a_{0}:=\inf _{x \in \mathbb{R}^{N}} a(x)>0$,

$\left(a_{2}\right)$ there exists an open bounded set $\Lambda \subset \mathbb{R}^{N}$ such that

$$
a_{0}<\min _{\partial \Lambda} a
$$

and $M:=\left\{x \in \Lambda: a(x)=a_{0}\right\} \neq \emptyset$.

Note that the local condition $\left(a_{2}\right)$ is weaker than (1.2) in the sense that it does not restrict the behavior of $a$ at infinity. This kind of hypothesis was introduced by Del Pino and Felmer [16] in the study of a semilinear Schrödinger equation.

We also suppose that $f \in C^{1}\left(\mathbb{R}^{+}, \mathbb{R}\right)$ satisfies

$\left(f_{1}\right) f(s)=o\left(s^{p-1}\right)$ as $s \rightarrow 0^{+}$,

$\left(f_{2}\right)$ there exists $p<q<p^{*}:=N p /(N-p)$ such that $f(s)=o\left(s^{q-1}\right)$ as $s \rightarrow \infty$,

$\left(f_{3}\right)$ there exists $p<\theta<q$ such that

$$
0<\theta F(s):=\theta \int_{0}^{s} f(\tau) \mathrm{d} \tau \leq s f(s) \text { for all } s>0,
$$


$\left(f_{4}\right)$ there exist $p<\sigma<p^{*}$ and $C_{\sigma}>0$ such that

$$
f^{\prime}(s) s-(p-1) f(s) \geq C_{\sigma} s^{\sigma-1} \quad \text { for all } s>0 .
$$

If $Y$ is a closed set of a topological space $X$, we denote by $\operatorname{cat}_{X}(Y)$ the LjusternikSchnirelmann category of $Y$ in $X$, namely the least number of closed and contractible sets in $X$ which cover $Y$. We are now ready to state the main result of this paper.

Theorem 1.1. Suppose that the potential a satisfies $\left(a_{1}\right)-\left(a_{2}\right)$ and the function $f$ satisfies $\left(f_{1}\right)-\left(f_{4}\right)$. Then, for any $\delta>0$ such that

$$
M_{\delta}:=\left\{x \in \mathbb{R}^{N}: \operatorname{dist}(x, M)<\delta\right\} \subset \Lambda,
$$

there exists $\varepsilon_{\delta}>0$ such that, for any $\varepsilon \in\left(0, \varepsilon_{\delta}\right)$, the problem $\left(P_{\varepsilon}\right)$ has at least cat $_{M_{\delta}}(M)$ solutions.

The proof of Theorem 1.1 will be done in three main steps. First, we apply the penalization method introduced by Del Pino and Felmer in [16]. It consists in modifying the function $f(u)$ outside the set $\Lambda$ in such a way that the energy functional of the modified problem satisfies the Palais-Smale condition.

In the second step, by using a technique due to Benci and Cerami [6], we relate the category of the set $M$ with the number of positive solutions of the modified problem. It is worthwhile to mention that, since we deal with a nonhomogeneous term $f(u)$, we cannot apply the concentration compactness principle [21] directly as in $[6,13,19]$. This difficulty is overcame by a detailed study of the energy functional restricted to its Nehari manifold.

In the last step we prove that the solutions obtained in the second one are in fact solutions of $\left(P_{\varepsilon}\right)$. The main problem here is that, since we are dealing with a quasilinear problem, we cannot argue as in $[16,25]$ to obtain uniform convergence (on compact sets) of the solutions. Thus, we proceed as in [12] by adapting the Moser's interaction method [22] in order to make careful estimates on the behavior of the solutions obtained in the second step.

To the best of our knowledge, there are no multiplicity results for quasilinear equations via penalization methods. However, our result seems to be new even in the semilinear case $p=2$. It extends the results in [19] and complements those of $[11,13,16,25]$.

We end this introduction by quoting some papers which dealt with the nonlinear Schrödinger problem

$$
\begin{cases}-\varepsilon^{2} \Delta u+V(x) u=K(x) u^{r-1}+Q(x) u^{t-1} & \text { in } \mathbb{R}^{N}, \\ u \in C^{2}\left(\mathbb{R}^{N}\right) \cap W^{1,2}\left(\mathbb{R}^{N}\right), u>0 & \text { in } \mathbb{R}^{N},\end{cases}
$$

with $\varepsilon>0,2<t<r<2^{*}, V \in C\left(\mathbb{R}^{N}\right)$ satisfying $V_{0}:=\inf _{\mathbb{R}^{N}} V>0, K$ positive and $K, Q \in L^{\infty}\left(\mathbb{R}^{N}\right)$. In [13], Cingolani and Lazzo considered $K \equiv 1, Q \equiv 0$ and 
$V$ verifying a global condition similar to (1.2). They related the number of solutions of (1.3) with the topology of the set where $V$ attains its minimum. Later, the same authors [14] supposed that $Q$ could change sign and obtained a multiplicity result involving the set of global minima of a function which gave the ground state levels of some autonomous problems related with (1.3). Roughly speaking, this function provides some kind of global median value between the minimum of $V$ and the maximum of $K$ and $Q$. We finally mention the paper of Ambrosetti, Malchiodi and Secchi [4], where the case $Q \equiv 0$ is studied. Among other results, they proved that the number of solutions of (1.3) is related with the set of minima of a function given explicitly in terms of $V, K, r$, and the dimension $N$. Since they used a finite dimensional reduction method, it is supposed that $V, K \in C^{2}\left(\mathbb{R}^{N}\right)$ are bounded, with $D^{2} V$ also bounded. We emphasize that, unlike the aforementioned works, we make no assumptions on the behavior of $a$ at infinity. In particular, we allow the potential $a$ be unbounded or $\liminf _{|x| \rightarrow \infty} a(x)<a_{0}$.

The paper is organized as follows: in Section 2, we modify the original problem and also prove some results concerning the autonomous problem related with $\left(P_{\varepsilon}\right)$. In Section 3, we present a multiplicity result for the modified problem. Theorem 1.1 is proved in Section 4.

\section{Some Notation and the Penalization Scheme}

Throughout the paper the conditions $\left(a_{1}\right)-\left(a_{2}\right)$ and $\left(f_{1}\right)-\left(f_{4}\right)$ will be assumed. Since we are interested in positive solutions, we extend $f$ to the whole real line by setting $f(s):=0$ for $s \leq 0$. To simplify notation, we write only $\int u$ instead of $\int_{\mathbb{R}^{N}} u(x) \mathrm{d} x$.

In order to overcome the lack of compactness of the problem $\left(P_{\varepsilon}\right)$ we make a slight adaptation of the penalization method introduced by Del Pino and Felmer in [16]. So, we choose $k>\theta /(\theta-p)$, where $\theta$ is given by $\left(f_{3}\right)$, and take $a>0$ to be the unique number such that $f(a) / a^{p-1}=1 / k$. We set

$$
\hat{f}(s):= \begin{cases}f(s) & \text { if } s \leq a, \\ \frac{1}{k} s^{p-1} & \text { if } s>a .\end{cases}
$$

Let $0<t_{a}<a<T_{a}$ and take a function $\eta \in C_{0}^{\infty}(\mathbb{R}, \mathbb{R})$ such that

$\left(\eta_{1}\right) \eta(s) \leq \hat{f}(s)$ for all $s \in\left[t_{a}, T_{a}\right]$,

$\left(\eta_{2}\right) \eta\left(t_{a}\right)=\hat{f}\left(t_{a}\right), \eta\left(T_{a}\right)=\hat{f}\left(T_{a}\right), \eta^{\prime}\left(t_{a}\right)=\hat{f}^{\prime}\left(t_{a}\right)$ and $\eta^{\prime}\left(T_{a}\right)=\hat{f}^{\prime}\left(T_{a}\right)$,

$\left(\eta_{3}\right)$ the map $s \mapsto \eta(s) / s^{p-1}$ is increasing for all $s \in\left[t_{a}, T_{a}\right]$.

By using the above functions we can define $\tilde{f} \in C^{1}(\mathbb{R}, \mathbb{R})$ as follows

$$
\tilde{f}(s):= \begin{cases}\hat{f}(s) & \text { if } s \notin\left[t_{a}, T_{a}\right], \\ \eta(s) & \text { if } s \in\left[t_{a}, T_{a}\right] .\end{cases}
$$


If $\chi_{\Lambda}$ denotes the characteristic function of the set $\Lambda$, we introduce the penalized nonlinearity $g: \mathbb{R}^{N} \times \mathbb{R} \rightarrow \mathbb{R}$ by setting

$$
g(x, s):=\chi_{\Lambda}(x) f(s)+\left(1-\chi_{\Lambda}(x)\right) \tilde{f}(s) .
$$

Notice that, using $\left(f_{1}\right)-\left(f_{4}\right)$ and $\left(\eta_{1}\right)-\left(\eta_{3}\right)$, it is easy to check that $g(x, s)$ satisfies the following properties:

$\left(g_{1}\right) g(x, s)=o\left(s^{p-1}\right)$ as $s \rightarrow 0$, uniformly in $x \in \mathbb{R}^{N}$,

$\left(g_{2}\right) g(x, s)=o\left(s^{q-1}\right)$ as $s \rightarrow \infty$, for some $q \in\left(p, p^{*}\right)$,

$\left(g_{3}\right)$ there exists $\theta \in(p, q)$ such that

(i) $0<\theta G(x, s):=\theta \int_{0}^{s} g(x, \tau) \mathrm{d} \tau<g(x, s) s$ for all $x \in \Lambda, s>0$,

(ii) $0 \leq p G(x, s) \leq g(x, s) s \leq s^{p} / k$ for all $x \in \mathbb{R}^{N} \backslash \Lambda, s>0$,

$\left(g_{4}\right)$ the function $s \mapsto g(x, s) / s^{p-1}$ is increasing for all $x \in \Lambda, s>0$.

Remark 2.1. It is easy to check that, if $u_{\varepsilon}$ is a positive solution of the equation

$$
-\varepsilon^{p} \operatorname{div}\left(a(x)|\nabla u|^{p-2} \nabla u\right)+u^{p-1}=g(x, u) \quad \text { in } \mathbb{R}^{N}
$$

such that $u_{\varepsilon}(x) \leq t_{a}$ for all $x \in \mathbb{R}^{N} \backslash \Lambda$, then $g\left(x, u_{\varepsilon}\right)=f\left(u_{\varepsilon}\right)$ and therefore $u_{\varepsilon}$ is also a solution of $\left(P_{\varepsilon}\right)$.

In view of the remark above, we deal in the sequel with the penalized problem

$\left(\tilde{P}_{\varepsilon}\right) \quad \begin{cases}-\operatorname{div}\left(a(\varepsilon x)|\nabla u|^{p-2} \nabla u\right)+u^{p-1}=g(\varepsilon x, u) & \text { in } \mathbb{R}^{N}, \\ u \in C_{\operatorname{loc}}^{1, \alpha}\left(\mathbb{R}^{N}\right) \cap W^{1, p}\left(\mathbb{R}^{N}\right), u(x)>0 & \text { for all } x \in \mathbb{R}^{N},\end{cases}$

and we will look for solutions $u_{\varepsilon}$ of $\left(\tilde{P}_{\varepsilon}\right)$ verifying

$$
u_{\varepsilon}(x) \leq t_{a} \quad \text { for all } x \in \mathbb{R}^{N} \backslash \Lambda_{\varepsilon},
$$

where

$$
\Lambda_{\varepsilon}:=\left\{x \in \mathbb{R}^{N}: \varepsilon x \in \Lambda\right\} .
$$

For any $\varepsilon>0$, we denote by $X_{\varepsilon}$ the Sobolev space $W^{1, p}\left(\mathbb{R}^{N}\right)$ endowed with the norm

$$
\|u\|_{\varepsilon}:=\left\{\int\left(a(\varepsilon x)|\nabla u|^{p}+|u|^{p}\right)\right\}^{1 / p} .
$$

The weak solutions of $\left(\tilde{P}_{\varepsilon}\right)$ are the positive critical points of the $C^{1}$-functional $E_{\varepsilon}: X_{\varepsilon} \rightarrow \mathbb{R}$ given by

$$
E_{\varepsilon}(u):=\frac{1}{p} \int\left(a(\varepsilon x)|\nabla u|^{p}+|u|^{p}\right)-\int G(\varepsilon x, u) .
$$


For any given $\xi>0$ there exists $C_{\xi}>0$ such that

$$
|g(x, s)| \leq \xi|s|^{p-1}+C_{\xi}|s|^{q-1} \quad \text { for all } x \in \mathbb{R}^{N}, s \in \mathbb{R} .
$$

Thus, if

$$
\mathcal{N}_{\varepsilon}:=\left\{u \in X_{\varepsilon} \backslash\{0\}:\left\langle E_{\varepsilon}^{\prime}(u), u\right\rangle=0\right\}
$$

denotes the Nehari manifold of $E_{\varepsilon}$, we can use (2.1) and $\left(g_{3}\right)$ to obtain $r_{\varepsilon}>0$ such that

$$
\|u\|_{\varepsilon} \geq r_{\varepsilon}>0 \quad \text { for all } u \in \mathcal{N}_{\varepsilon} .
$$

In what follows, supp $u$ denotes the support of a function $u$.

Lemma 2.2. Let $u \in X_{\varepsilon}$ be a nonnegative function such that $\operatorname{supp} u \cap \Lambda_{\varepsilon}$ has positive measure. Then there exists a unique $t_{u}>0$ such that $t_{u} u \in \mathcal{N}_{\varepsilon}$.

Proof. If $\psi(t):=E_{\varepsilon}(t u)$ for $t \geq 0$, inequality (2.4) and the Sobolev embeddings imply that $\psi$ is positive near $t=0$. Moreover,

$$
\psi(t) \leq \frac{t^{p}}{p}\|u\|_{\varepsilon}^{p}-\int_{\Lambda_{\varepsilon}} G(\varepsilon x, t u) .
$$

Since the set $\left\{x \in \Lambda_{\varepsilon}: u(x)>0\right\}$ has positive measure, the above expression and $\left(g_{3}\right)(\mathrm{i})$ imply that $\psi(t) \rightarrow-\infty$ as $t \rightarrow \infty$. Hence, there exists $t_{u}>0$ such that $\psi^{\prime}\left(t_{u}\right)=0$, namely the point where $\psi$ attains its maximum. A direct computation shows that $t_{u} u \in \mathcal{N}_{\varepsilon}$. The uniqueness follows from the monotonicity condition $\left(g_{4}\right)$. The lemma is proved.

Remark 2.3. If $u \in \mathcal{N}_{\varepsilon}$, then the last inequality in $\left(g_{3}\right)$ (ii) implies that supp $u \cap \Lambda_{\varepsilon}$ has positive measure. Thus, we can argue as above to conclude that $E_{\varepsilon}(t u) \leq E_{\varepsilon}(u)$ for all $t \geq 0$.

We make now some comments about the autonomous problem

$$
\begin{cases}-a_{0} \operatorname{div}\left(|\nabla u|^{p-2} \nabla u\right)+u^{p-1}=f(u) & \text { in } \mathbb{R}^{N}, \\ u \in C_{\operatorname{loc}}^{1, \alpha}\left(\mathbb{R}^{N}\right) \cap W^{1, p}\left(\mathbb{R}^{N}\right), u(x)>0 & \text { for all } x \in \mathbb{R}^{N},\end{cases}
$$

whose solutions are the positive critical points of the $C^{1}$-functional $I_{0}: W^{1, p}\left(\mathbb{R}^{N}\right) \rightarrow$ $\mathbb{R}$ given by

$$
I_{0}(u):=\frac{1}{p}\|u\|_{X}^{p}-\int F(u),
$$

where

$$
\|u\|_{X}:=\left\{\int\left(a_{0}|\nabla u|^{p}+|u|^{p}\right)\right\}^{1 / p} \quad \text { for all } u \in X:=W^{1, p}\left(\mathbb{R}^{N}\right) .
$$


Let

$$
\mathcal{M}_{0}:=\left\{u \in X \backslash\{0\}:\left\langle I_{0}^{\prime}(u), u\right\rangle=0\right\}
$$

be the Nehari manifold of $I_{0}$ and consider the minimization problem

$$
m_{0}:=\inf _{u \in \mathcal{M}_{0}} I_{0}(u)
$$

It can be proved (see [28, Chapter 4]) that $m_{0}$ is positive and can be characterized as

$$
m_{0}=\inf _{u \in X \backslash\{0\}} \sup _{t \geq 0} I_{0}(t u)>0 .
$$

Moreover, for any $u \in X \backslash\{0\}$, there exists a unique $t_{u}>0$ such that $t_{u} u \in \mathcal{M}_{0}$. The maximum of the function $t \mapsto I_{0}(t u)$ for $t \geq 0$ is achieved at $t=t_{u}$.

The following result presents an interesting property of the minimizing sequences of $m_{0}$.

Lemma 2.4. Let $\left(u_{n}\right) \subset \mathcal{M}_{0}$ be such that $I_{0}\left(u_{n}\right) \rightarrow m_{0}$ and $u_{n} \rightarrow u$ weakly in $X$. Then there exists a sequence $\left(\bar{y}_{n}\right) \subset \mathbb{R}^{N}$ such that $v_{n}:=u_{n}\left(\cdot+\bar{y}_{n}\right) \rightarrow v \in \mathcal{M}_{0}$ with $I_{0}(v)=m_{0}$. Moreover, if $u \neq 0$, then $\left(\tilde{y}_{n}\right)$ can be taken identically zero and therefore $u_{n} \rightarrow u$ in $X$.

Proof. The proof is similar to that presented in [2, Theorem 3.1] and it will be omitted.

We recall that a solution $u$ of (2.6) is called ground state solution if

$$
I_{0}(u)=\min \left\{I_{0}(v): v \text { is a solution of (2.6) }\right\} .
$$

As an easy consequence of the above lemma we have the following useful result.

Corollary 2.5. The problem (2.6) possesses a ground state solution.

Proof. By the preceding lemma, the number $m_{0}$ is achieved by some $u \in$ $\mathcal{M}_{0}$. Since $u$ is a critical point of the functional $I_{0}$ restricted to $\mathcal{M}_{0}$, we can use standard arguments (see [2, Proposition 3.2] for example) to get $I_{0}^{\prime}(u)=0$ in the dual space of $X$. Hence, $u$ satisfies the equation in (2.6). If we denote by $u^{-}:=\max \{-u, 0\}$ the negative part of $u$, we get

$$
0=\left\langle I_{0}^{\prime}(u), u^{-}\right\rangle=\left\|u^{-}\right\|_{X}^{p}-\int f(u) u^{-}=\left\|u^{-}\right\|_{X}^{p}
$$

and therefore $u \geq 0$ in $\mathbb{R}^{N}$. Adapting arguments from [20, Theorem 1.11] we conclude that $u \in L^{\infty}\left(\mathbb{R}^{N}\right) \cap C_{\text {loc }}^{1, \alpha}\left(\mathbb{R}^{N}\right)$ for some $0<\alpha<1$, and therefore it follows from Harnack's inequality [27] that $u(x)>0$ for all $x \in \mathbb{R}^{N}$. Since all critical points of $I_{0}$ belong to $\mathcal{M}_{0}$, the solution $u$ is a ground state solution. 


\section{Multiplicity of Solutions for the Modified Problem}

We devote this section to the proof of the following result.

Theorem 3.1. For any $\delta>0$ such that $M_{\delta} \subset \Lambda$, there exists $\varepsilon_{\delta}>0$ such that, for any $\varepsilon \in\left(0, \varepsilon_{\delta}\right)$, the problem $\left(\tilde{P}_{\varepsilon}\right)$ has at least $\mathrm{cat}_{M_{\delta}}(M)$ solutions.

Since we are intending to apply critical point theory we need to introduce some compactness property. So, let $V$ be a Banach space, $V$ be a $C^{1}$-manifold of $V$ and $I: V \rightarrow \mathbb{R}$ a $C^{1}$-functional. We say that $I \mid \mathcal{V}$ satisfies the Palais-Smale condition at level $c\left((\mathrm{PS})_{c}\right.$ for short) if any sequence $\left(u_{n}\right) \subset \mathcal{V}$ such that $I\left(u_{n}\right) \rightarrow$ $c$ and $\left\|I^{\prime}\left(u_{n}\right)\right\|_{*} \rightarrow 0$ contains a convergent subsequence. Here, we are denoting by $\left\|I^{\prime}(u)\right\|_{*}$ the norm of the derivative of $I$ restricted to $\mathcal{V}$ at the point $u$.

We will use the following Ljusternik-Schnirelmann abstract result for $C^{1}$ manifolds (see [18, Corollary 4.17]).

Theorem 3.2. Let I be a $C^{1}$-functional defined on a $C^{1}$-Finsler manifold $\mathcal{V}$. If $I$ is bounded below and satisfies the Palais-Smale condition, then I has at least cat $(\mathcal{V})$ distinct critical points.

The proof of Theorem 3.1 is rather long and will be done by applying the above result to the functional $E_{\varepsilon}$ restricted to $\mathcal{N}_{\varepsilon}$. Thus, we need first relate the category of $\mathcal{N}_{\varepsilon}$ with that of $M$. This is exactly the content of the next two subsections. The following result, whose proof is similar to that presented in [7, Lemma 4.3], will be used.

Lemma 3.3. Let $H, \Omega^{+}, \Omega^{-}$be closed sets with $\Omega^{-} \subset \Omega^{+}$. Let $\beta: H \rightarrow \Omega^{+}$, $\Phi: \Omega^{-} \rightarrow H$ be two continuous maps such that $\beta \circ \Phi$ is homotopically equivalent to the embedding $\iota: \Omega^{-} \rightarrow \Omega^{+}$. Then $\operatorname{cat}(H) \geq \operatorname{cat}_{\Omega^{+}}\left(\Omega^{-}\right)$.

3.1. The $\operatorname{map} \Phi_{\varepsilon}$ Let $\delta>0$ be such that $M_{\delta} \subset \Lambda$ and choose a cut-off function $\psi \in C_{0}^{\infty}\left(\mathbb{R}^{+},[0,1]\right)$ such that $\psi(s):=1$ if $0 \leq s \leq \delta / 2$ and $\psi(s):=0$ if $s \geq \delta$. If $\omega$ is a ground-state solution of the problem (2.6), we define for each $y \in M$ the function

$$
\Psi_{\varepsilon, y}(x):=\psi(|\varepsilon x-y|) \omega\left(\frac{\varepsilon x-y}{\varepsilon}\right) .
$$

Let $\Phi_{\varepsilon}: M \rightarrow \mathcal{N}_{\varepsilon}$ be given by

$$
\Phi_{\varepsilon}(y):=t_{\varepsilon} \Psi_{\varepsilon, y},
$$

where $t_{\varepsilon}>0$ is the unique number such that $t_{\varepsilon} \Psi_{\varepsilon, y} \in \mathcal{N}_{\varepsilon}$. Since $\psi(|\varepsilon x-y|) \equiv 1$ in $B_{\delta / 2 \varepsilon}(y / \varepsilon)$ and $y / \varepsilon \in \Lambda_{\varepsilon}$, Lemma 2.2 shows that $\Phi_{\varepsilon}$ is well defined.

Lemma 3.4. Uniformly for $y \in M$, we have

$$
\lim _{\varepsilon \rightarrow 0^{+}} E_{\varepsilon}\left(\Phi_{\varepsilon}(y)\right)=m_{0} .
$$


Proof. Arguing by contradiction, we suppose that there exist $\gamma>0,\left(y_{n}\right) \subset$ $M$ and $\varepsilon_{n} \rightarrow 0^{+}$such that

$$
\left|E_{\varepsilon_{n}}\left(\Phi_{\varepsilon_{n}}\left(y_{n}\right)\right)-m_{0}\right| \geq \gamma>0 .
$$

In order to simplify the notation, we write only $\Phi_{n}, \Psi_{n}$ and $t_{n}$ to denote $\Phi_{\varepsilon_{n}}\left(y_{n}\right)$, $\Psi_{\varepsilon_{n}, y_{n}}$ and $t_{\varepsilon_{n}}$, respectively.

Since $\left\langle E_{\varepsilon_{n}}^{\prime}\left(t_{n} \Psi_{n}\right), t_{n} \Psi_{n}\right\rangle=0$, with the change of variables $z:=\left(\varepsilon_{n} x-\right.$ $\left.y_{n}\right) / \varepsilon_{n}$ we get

$$
\begin{aligned}
\left\|t_{n} \Psi_{n}\right\|_{\varepsilon_{n}}^{p} & =\int g\left(\varepsilon_{n} x, t_{n} \Psi_{n}(x)\right) t_{n} \Psi_{n}(x) \mathrm{d} x \\
& =\int g\left(\varepsilon_{n} z+y_{n}, t_{n} \psi\left(\left|\varepsilon_{n} z\right|\right) w(z)\right) t_{n} \psi\left(\left|\varepsilon_{n} z\right|\right) w(z) \mathrm{d} z .
\end{aligned}
$$

Notice that, if $z \in B_{\delta / \varepsilon_{n}}(0)$, then $\varepsilon_{n} z+y_{n} \in B_{\delta}\left(y_{n}\right) \subset M_{\delta} \subset \Lambda$. Recalling that $g(x, s)=f(s)$ for any $x \in \Lambda$ and $\psi(s)=0$ for $s \geq \delta$, the above expression yields

$$
\left\|\Psi_{n}\right\|_{\varepsilon_{n}}^{p}=\int \frac{f\left(t_{n} \psi\left(\left|\varepsilon_{n} z\right|\right) \omega(z)\right)}{\left(t_{n} \psi\left(\left|\varepsilon_{n} z\right|\right) \omega(z)\right)^{p-1}}\left|\psi\left(\left|\varepsilon_{n} z\right|\right) \omega(z)\right|^{p} \mathrm{~d} z .
$$

Let $\alpha:=\min \{w(z):|z| \leq \delta / 2\}$. If $n_{0} \in \mathbb{N}$ is such that $B_{\delta / 2}(0) \subset B_{\delta /\left(2 \varepsilon_{n}\right)}$ for all $n \geq n_{0}$, we obtain

$$
\begin{aligned}
\left\|\Psi_{n}\right\|_{\varepsilon_{n}}^{p} & \geq \int_{B_{\delta / 2}(0)} \frac{f\left(t_{n} \omega(z)\right)}{\left(t_{n} \omega(z)\right)^{p-1}}|\omega(z)|^{p} \mathrm{~d} z \\
& \geq \frac{f\left(t_{n} \alpha\right)}{\left(t_{n} \alpha\right)^{p-1}} \int_{B_{\delta / 2}(0)}|\omega(z)|^{p} \mathrm{~d} z,
\end{aligned}
$$

for all $n \geq n_{0}$, where we have used that $s \mapsto f(s) / s^{p-1}$ is increasing (see $\left(f_{4}\right)$ ). By using Lebesgue's theorem we may easily check that

$$
\left\|\Psi_{n}\right\|_{\varepsilon_{n}}^{p} \rightarrow\|\omega\|_{X}^{p} \quad \text { and } \quad \int F\left(\Psi_{n}\right) \rightarrow \int F(\omega) .
$$

If $\left|t_{n}\right| \rightarrow \infty$, we can use (3.3) and $\left(f_{3}\right)$ to conclude that $\left\|\Psi_{n}\right\|_{\varepsilon_{n}}^{p} \rightarrow+\infty$, contradicting the first assertion above. Thus, up to a subsequence, $t_{n} \rightarrow t_{0} \geq 0$.

For any given $\xi>0$, we can use (2.4) and $t_{n} \Psi_{n} \in \mathcal{N}_{\varepsilon_{n}}$ to get

$$
\left\|\Psi_{n}\right\|_{\varepsilon_{n}}^{p} \leq \xi \int\left|\Psi_{n}\right|^{p}+C_{\xi} t_{n}^{q-p} \int\left|\Psi_{n}\right|^{q} .
$$

Since $\xi$ is arbitrary, if $t_{0}=0$ the above expression and the boundedness of ( $\left.\Psi_{n}\right)$ would imply $\left\|\Psi_{n}\right\|_{\varepsilon_{n}}^{p} \rightarrow 0$, contradicting (3.4). Hence $t_{0}>0$ and we can take the 
limit in (3.2) to obtain

$$
\int\left(a_{0}|\nabla \omega|^{p}+|\omega|^{p}\right)=\int \frac{f\left(t_{0} \omega\right) \omega}{t_{0}^{p-1}}
$$

from which it follows that $t_{0} \omega \in \mathcal{M}_{0}$. Since $\omega$ also belongs to $\mathcal{M}_{0}$, we conclude that $t_{0}=1$. Thus, letting $n \rightarrow \infty$ in

$$
\begin{aligned}
E_{\varepsilon_{n}}\left(\Phi_{n}\right)=\frac{t_{n}^{p}}{p} \int\left(a\left(\varepsilon_{n} z+y_{n}\right)\left|\nabla\left(\psi\left(\left|\varepsilon_{n} z\right|\right) \omega(z)\right)\right|^{p}\right. & \left.+\left|\psi\left(\left|\varepsilon_{n} z\right|\right) \omega(z)\right|^{p}\right) \mathrm{d} z \\
& -\int F\left(t_{n} \psi\left(\left|\varepsilon_{n} z\right|\right) \omega(z)\right) \mathrm{d} z
\end{aligned}
$$

and using the second statement in (3.4), we get

$$
\lim _{n \rightarrow \infty} E_{\varepsilon_{n}}\left(\Phi_{\varepsilon_{n}}\left(y_{n}\right)\right)=I_{0}(\omega)=m_{0}
$$

which contradicts (3.1) and proves the lemma.

3.2. The $\operatorname{map} \beta_{\varepsilon} \quad$ Let $\delta>0$ be such that $M_{\delta} \subset \Lambda$ and choose $\rho=\rho_{\delta}>0$ in such a way that $M_{\delta} \subset B_{\rho}(0)$. Let $Y: \mathbb{R}^{N} \rightarrow \mathbb{R}^{N}$ be defined as $Y(x):=x$ for $|x|<\rho$ and $\Upsilon(x):=\rho x /|x|$ for $|x| \geq \rho$. Finally, consider the barycenter map $\beta_{\varepsilon}: \mathcal{N}_{\varepsilon} \rightarrow \mathbb{R}^{N}$ given by

$$
\beta_{\varepsilon}(u):=\frac{\int \Upsilon(\varepsilon x)|u(x)|^{p} \mathrm{~d} x}{\int|u(x)|^{p} \mathrm{~d} x} .
$$

Since $M \subset B_{\rho}(0)$, we can use the definition of $\Upsilon$ and Lebesgue's theorem to conclude that

$$
\lim _{\varepsilon \rightarrow 0} \beta_{\varepsilon}\left(\Phi_{\varepsilon}(y)\right)=y \quad \text { uniformly for } y \in M .
$$

Lemma 3.5. Let $\left(\varepsilon_{n}\right) \subset \mathbb{R}^{+}$be such that $\varepsilon_{n} \rightarrow 0$ and $\left(u_{n}\right) \subset \mathcal{N}_{\varepsilon_{n}}$ satisfying $E_{\varepsilon_{n}}\left(u_{n}\right) \rightarrow m_{0}$. Then there exists a sequence $\left(\tilde{y}_{n}\right) \subset \mathbb{R}^{N}$ such that the sequence $v_{n}:=u_{n}\left(\cdot+\bar{y}_{n}\right)$ has a convergent subsequence in $W^{1, p}\left(\mathbb{R}^{N}\right)$. Moreover, up to a subsequence, $\left(y_{n}\right):=\left(\varepsilon_{n} \tilde{y}_{n}\right)$ satisfies $y_{n} \rightarrow y \in M$.

Proof. We start by proving that there exists a sequence $\left(\tilde{y}_{n}\right) \subset \mathbb{R}^{N}$ and constants $R, \gamma>0$ such that

$$
\liminf _{n \rightarrow \infty} \int_{B_{R}\left(\tilde{y}_{n}\right)}\left|u_{n}\right|^{p} \geq \gamma>0 .
$$


Indeed, if this is not true, then the boundedness of $\left(u_{n}\right)$ in $X$ and a lemma due to P.-L. Lions [21, Lemma I.1] imply that $u_{n} \rightarrow 0$ in $L^{s}\left(\mathbb{R}^{N}\right)$ for all $p<s<p^{*}$. Given $\xi>0$, we can use (2.4) and $u_{n} \in \mathcal{N}_{\varepsilon_{n}}$ to get

$$
\left\|u_{n}\right\|_{\varepsilon_{n}}^{p}=\int g\left(\varepsilon x, u_{n}\right) u_{n} \leq \xi \int\left|u_{n}\right|^{p}+C_{\xi} \int\left|u_{n}\right|^{q} .
$$

Since $u_{n} \rightarrow 0$ in $L^{q}\left(\mathbb{R}^{N}\right)$ and $\xi$ is arbitrary, we conclude that $\left\|u_{n}\right\|_{\varepsilon_{n}} \rightarrow 0$. Moreover, since $\int g\left(\varepsilon \mathcal{X}, u_{n}\right) u_{n} \rightarrow 0$, it follows from $\left(g_{3}\right)$ that $\int G\left(\varepsilon \mathcal{X}, u_{n}\right) \rightarrow 0$. Hence, $E_{\varepsilon_{n}}\left(u_{n}\right) \rightarrow 0$, contradicting $m_{0}>0$. Thus, (3.6) holds and, along a subsequence,

$$
v_{n}:=u_{n}\left(\cdot+\bar{y}_{n}\right)-v \neq 0 \quad \text { weakly in } X .
$$

Let $\left(t_{n}\right) \subset \mathbb{R}^{+}$be such that $w_{n}:=t_{n} v_{n} \in \mathcal{M}_{0}$. Defining $y_{n}:=\varepsilon_{n} \tilde{y}_{n}$, changing variables and using $u_{n} \in \mathcal{N}_{\varepsilon_{n}}$, we get

$$
\begin{aligned}
m_{0} \leq I_{0}\left(w_{n}\right) & =\frac{t_{n}^{p}}{p} \int a_{0}\left|\nabla v_{n}\right|^{p}+\frac{t_{n}^{p}}{p} \int\left|v_{n}\right|^{p}-\int F\left(t_{n} v_{n}\right) \\
& \leq \frac{t_{n}^{p}}{p} \int\left(a\left(\varepsilon_{n} x\right)\left|\nabla u_{n}\right|^{p}+\left|u_{n}\right|^{p}\right)-\int G\left(\varepsilon_{n} x, t_{n} u_{n}\right) \\
& =E_{\varepsilon_{n}}\left(t_{n} u_{n}\right) \leq E_{\varepsilon_{n}}\left(u_{n}\right) .
\end{aligned}
$$

Hence $\lim _{n \rightarrow \infty} I_{0}\left(w_{n}\right)=m_{0}$, from which it follows that $w_{n} \nmid 0$ in $X$.

Since $\left(w_{n}\right)$ and $\left(v_{n}\right)$ are bounded in $X$ and $v_{n}+0$ in $X$, the sequence $\left(t_{n}\right)$ is bounded. Thus, up to a subsequence, $t_{n} \rightarrow t_{0} \geq 0$. If $t_{0}=0$ then $\left\|w_{n}\right\|_{X} \rightarrow 0$, which does not make sense. Hence $t_{0}>0$, and therefore the sequence $\left(w_{n}\right)$ satisfies

$$
I_{0}\left(w_{n}\right) \rightarrow m_{0}, w_{n} \rightarrow w:=t_{0} v \neq 0 \quad \text { weakly in } X .
$$

It follows from Lemma 2.4 that $w_{n} \rightarrow w$, or equivalently, $v_{n} \rightarrow v$ in $X$. This proves the first part of the lemma.

We claim that $\left(y_{n}\right)$ has a bounded subsequence. Indeed, if this is not the case, then $\left|y_{n}\right| \rightarrow \infty$. Consider $R>0$ such that $\Lambda \subset B_{R}(0)$. Since we may suppose that $\left|y_{n}\right|>2 R$, for any $z \in B_{R / \varepsilon_{n}}(0)$ we have

$$
\left|\varepsilon_{n} z+y_{n}\right| \geq\left|y_{n}\right|-\left|\varepsilon_{n} z\right|>R \text {. }
$$

If $\Gamma_{n}:=B_{R / \varepsilon_{n}}(0)$, we can use $\left(u_{n}\right) \subset \mathcal{N}_{\varepsilon_{n}},\left(a_{1}\right)$, the change of variables $x \mapsto$ $z+\tilde{y}_{n}$, the expression above, and (2.1) to get 


$$
\begin{aligned}
\int\left(a_{0}\left|\nabla v_{n}\right|^{p}+\left|v_{n}\right|^{p}\right) \mathrm{d} z & \leq \int g\left(\varepsilon_{n} z+y_{n}, v_{n}\right) v_{n} \mathrm{~d} z \\
& =\int_{\Gamma_{n}} \tilde{f}\left(v_{n}\right) v_{n} \mathrm{~d} z+\int_{\mathbb{R}^{N} \backslash \Gamma_{n}} g\left(\varepsilon_{n} z+y_{n}, v_{n}\right) v_{n} \mathrm{~d} z \\
& \leq \int_{\Gamma_{n}} \tilde{f}\left(v_{n}\right) v_{n} \mathrm{~d} z+\int_{\mathbb{R}^{N} \backslash \Gamma_{n}} f\left(v_{n}\right) v_{n} \mathrm{~d} z .
\end{aligned}
$$

Since $\tilde{f}(s) \leq(1 / k) s^{p-1}$, the above expression, $v_{n} \rightarrow v$ in $X$, and the Lebesgue Dominated Convergence theorem imply that

$$
\left(1-\frac{1}{k}\right)\left\|v_{n}\right\|_{X}^{p} \leq \int_{\mathbb{R}^{N} \backslash B_{R / \varepsilon_{n}}(0)} f\left(v_{n}\right) v_{n} \mathrm{~d} z=o_{n}(1) .
$$

Letting $n \rightarrow \infty$ we conclude that $v=0$, which is a contradiction. Thus, up to a subsequence, $y_{n} \rightarrow y \in \mathbb{R}^{N}$.

It remains to check that $y \in M$. Arguing by contradiction again, we suppose that $a(y)>a_{0}$. Then, recalling that $w_{n} \rightarrow w$, we can use Fatou's lemma to obtain

$$
\begin{aligned}
m_{0} & =I_{0}(w)<\frac{1}{p} \int\left(a(y)|\nabla w|^{p}+|w|^{p}\right)-\int F(w) \\
& \leq \liminf _{n \rightarrow \infty}\left\{\frac{1}{p} \int\left(a\left(\varepsilon_{n} z+y_{n}\right)\left|\nabla w_{n}\right|^{p}+\left|w_{n}\right|^{p}\right)-\int F\left(w_{n}\right)\right\} \\
& \leq \liminf _{n \rightarrow \infty} E_{\varepsilon_{n}}\left(t_{n} u_{n}\right) \leq \liminf _{n \rightarrow \infty} E_{\varepsilon_{n}}\left(u_{n}\right)=m_{0},
\end{aligned}
$$

which does not make sense. The proof is finished.

Following [13], we introduce the set

$$
\Sigma_{\varepsilon}:=\left\{u \in \mathcal{N}_{\varepsilon}: E_{\varepsilon}(u) \leq m_{0}+h(\varepsilon)\right\},
$$

where $h: \mathbb{R}^{+} \rightarrow \mathbb{R}^{+}$is such that $h(\varepsilon) \rightarrow 0$ as $\varepsilon \rightarrow 0^{+}$. Given $y \in M$, we can use Lemma 3.4 to conclude that $h(\varepsilon)=\left|E_{\varepsilon}\left(\Phi_{\varepsilon}(y)\right)-m_{0}\right|$ is such that $h(\varepsilon) \rightarrow 0$ as $\varepsilon \rightarrow 0^{+}$. Thus, $\Phi_{\varepsilon}(y) \in \Sigma_{\varepsilon}$ and therefore $\Sigma_{\varepsilon} \neq \emptyset$ for any $\varepsilon>0$.

Lemma 3.6. For any $\delta>0$ we have that

$$
\lim _{\varepsilon \rightarrow 0^{+}} \sup _{u \in \Sigma_{\varepsilon}} \operatorname{dist}\left(\beta_{\varepsilon}(u), M_{\delta}\right)=0 .
$$

Proof. Let $\left(\varepsilon_{n}\right) \subset \mathbb{R}^{+}$be such that $\varepsilon_{n} \rightarrow 0$. By definition there exists $\left(u_{n}\right) \subset$ $\sum_{\varepsilon_{n}}$ such that

$$
\operatorname{dist}\left(\beta_{\varepsilon_{n}}\left(u_{n}\right), M_{\delta}\right)=\sup _{u \in \Sigma_{\varepsilon_{n}}} \operatorname{dist}\left(\beta_{\varepsilon_{n}}(u), M_{\delta}\right)+o_{n}(1),
$$


where hereafter $o_{n}(1)$ denotes a quantity which goes to 0 as $n \rightarrow \infty$. Thus, it suffices to find a sequence $\left(y_{n}\right) \subset M_{\delta}$ such that

$$
\left|\beta_{\varepsilon_{n}}\left(u_{n}\right)-y_{n}\right|=o_{n}(1) \text {. }
$$

In order to obtain such a sequence, we note that $I_{0}\left(t u_{n}\right) \leq E_{\varepsilon_{n}}\left(t u_{n}\right)$ for any $t \geq 0$. Thus, recalling that $\left(u_{n}\right) \subset \Sigma_{\varepsilon_{n}} \subset \mathcal{N}_{\varepsilon_{n}}$, we can use (2.7) to get

$$
m_{0} \leq \max _{t \geq 0} I_{0}\left(t u_{n}\right) \leq \max _{t \geq 0} E_{\varepsilon_{n}}\left(t u_{n}\right)=E_{\varepsilon_{n}}\left(u_{n}\right) \leq m_{0}+h\left(\varepsilon_{n}\right),
$$

from which it follows that $E_{\varepsilon_{n}}\left(u_{n}\right) \rightarrow m_{0}$. Thus, we may invoke Lemma 3.5 to obtain a sequence $\left(\bar{y}_{n}\right) \subset \mathbb{R}^{N}$ such that $\left(y_{n}\right):=\left(\varepsilon_{n} \bar{y}_{n}\right) \subset M_{\delta}$ for $n$ sufficiently large. Hence,

$$
\begin{aligned}
\beta_{\varepsilon_{n}}\left(u_{n}\right) & =\frac{\int \Upsilon\left(\varepsilon_{n} x\right)\left|u_{n}\right|^{p} \mathrm{~d} x}{\int\left|u_{n}\right|^{p} \mathrm{~d} x}=\frac{\int \Upsilon\left(\varepsilon_{n} z+y_{n}\right)\left|u_{n}\left(z+\bar{y}_{n}\right)\right|^{p} \mathrm{~d} z}{\int\left|u_{n}\left(z+\bar{y}_{n}\right)\right|^{p} \mathrm{~d} z} \\
& =y_{n}+\frac{\int\left(\Upsilon\left(\varepsilon_{n} z+y_{n}\right)-y_{n}\right)\left|u_{n}\left(z+\bar{y}_{n}\right)\right|^{p} \mathrm{~d} z}{\int\left|u_{n}\left(z+\bar{y}_{n}\right)\right|^{p} \mathrm{~d} z} .
\end{aligned}
$$

Since $\varepsilon_{n} z+y_{n} \rightarrow y \in M$, we have that $\beta_{\varepsilon_{n}}\left(u_{n}\right)=y_{n}+o_{n}(1)$ and therefore the sequence $\left(y_{n}\right)$ verifies (3.9). The lemma is proved.

3.3. Proof of Theorem 3.1 In view of condition $\left(g_{3}\right)$, it is standard to check that $E_{\varepsilon}$ satisfies the Palais-Smale condition at any level (see [25, Lemma 3.1] for example). The next result shows that the same is true for $E_{\varepsilon}$ constrained to the manifold $\mathcal{N}_{\varepsilon}$.

Lemma 3.7. The functional $E_{\varepsilon}$ restricted to $\mathcal{N}_{\varepsilon}$ satisfies the Palais-Smale condition.

Proof. Let $\left(u_{n}\right) \subset \mathcal{N}_{\varepsilon}$ be such that $E_{\varepsilon}\left(u_{n}\right) \rightarrow c$ and $\left\|E_{\varepsilon}^{\prime}\left(u_{n}\right)\right\|_{*} \rightarrow 0$. Then there exists $\left(\lambda_{n}\right) \subset \mathbb{R}$ such that

$$
E_{\varepsilon}^{\prime}\left(u_{n}\right)=\lambda_{n} J_{\varepsilon}^{\prime}\left(u_{n}\right)+o_{n}(1),
$$

where $J_{\varepsilon}: X_{\varepsilon} \rightarrow \mathbb{R}$ is given by

$$
J_{\varepsilon}(u):=\int a(\varepsilon x)|\nabla u|^{p}+\int|u|^{p}-\int g(\varepsilon x, u) u .
$$


Since $\left(u_{n}\right) \subset \mathcal{N}_{\varepsilon}$, we can use $(2.1),\left(\eta_{3}\right)$ and $\left(f_{4}\right)$ to get

$$
\begin{aligned}
\left\langle J_{\varepsilon}^{\prime}\left(u_{n}\right), u_{n}\right\rangle= & \int\left\{(p-1) g\left(\varepsilon x, u_{n}\right) u_{n}-g^{\prime}\left(\varepsilon x, u_{n}\right) u_{n}^{2}\right\} \\
= & \int_{\Lambda_{\varepsilon} \cup\left\{u_{n}<t_{a}\right\}}\left\{(p-1) f\left(u_{n}\right) u_{n}-f^{\prime}\left(u_{n}\right) u_{n}^{2}\right\} \\
& \quad+\int_{\left(\mathbb{R}^{N} \backslash \Lambda_{\varepsilon}\right) \cap\left\{t_{a} \leq u_{n} \leq T_{a}\right\}}\left\{(p-1) \eta\left(u_{n}\right) u_{n}-\eta^{\prime}\left(u_{n}\right) u_{n}^{2}\right\} \\
\leq & -C_{\sigma} \int_{\Lambda_{\varepsilon} \cup\left\{u_{n}<t_{a}\right\}}\left|u_{n}\right|^{\sigma} \leq-C_{\sigma} \int_{\Lambda_{\varepsilon}}\left|u_{n}\right|^{\sigma},
\end{aligned}
$$

where $g^{\prime}(x, s)$ means the derivative with respect to the second variable and the numbers $t_{a}$ and $T_{a}$ were fixed at the beginning of Section 2 .

By the above expression, we may suppose that $\left\langle J_{\varepsilon}^{\prime}\left(u_{n}\right), u_{n}\right\rangle \rightarrow \ell \leq 0$. We claim that $\ell<0$. If this is the case, it follows from

$$
0=\left\langle E_{\varepsilon}^{\prime}\left(u_{n}\right), u_{n}\right\rangle=\lambda_{n}\left\langle J_{\varepsilon}^{\prime}\left(u_{n}\right), u_{n}\right\rangle+o_{n}(1)
$$

that $\lambda_{n} \rightarrow 0$. Hence, use can use (3.10) to conclude that $E_{\varepsilon}^{\prime}\left(u_{n}\right) \rightarrow 0$ in the dual space of $X_{\varepsilon}$. Since we already know that the unconstrained functional satisfies Palais-Smale, we conclude that $\left(u_{n}\right)$ has a convergent subsequence.

It remains to prove that $\ell<0$. Suppose, by contradiction, that $\ell=0$. Then it follows from $\left|\left\langle J_{\varepsilon}^{\prime}\left(u_{n}\right), u_{n}\right\rangle\right| \geq C_{\sigma} \int_{\Lambda_{\varepsilon}}\left|u_{n}\right|^{\sigma}$ that $u_{n} \rightarrow 0$ in $L^{\sigma}\left(\Lambda_{\varepsilon}\right)$. By interpolation, $u_{n} \rightarrow 0$ in $L^{q}\left(\Lambda_{\varepsilon}\right)$. The same argument employed in the proof of Lemma 3.5 shows that $\int_{\Lambda_{\varepsilon}} g\left(\varepsilon x, u_{n}\right) u_{n}=o_{n}(1)$. This and $\left(g_{3}\right)($ ii) provide

$$
\left\|u_{n}\right\|_{\varepsilon}^{p}=\int_{\mathbb{R}^{N} \backslash \Lambda_{\varepsilon}} g\left(\varepsilon x, u_{n}\right) u_{n}+\int_{\Lambda_{\varepsilon}} g\left(\varepsilon x, u_{n}\right) u_{n} \leq \frac{1}{k} \int_{\mathbb{R}^{N} \backslash \Lambda_{\varepsilon}}\left|u_{n}\right|^{p}+o_{n}(1),
$$

and therefore

$$
\left(1-\frac{1}{k}\right)\left\|u_{n}\right\|_{\varepsilon}^{p}=o_{n}(1),
$$

which contradicts (2.5) and proves the lemma.

We are now ready to present the proof of Theorem 3.1.

Proof of Theorem 3.1. Given $\delta>0$ such that $M_{\delta} \subset \Lambda$, we can use (3.5), Lemmas 3.4 and 3.6, and argue as in [13, Section 6] to obtain $\varepsilon_{\delta}>0$ such that, for any $\varepsilon \in\left(0, \varepsilon_{\delta}\right)$, the diagram

$$
M \stackrel{\Phi_{\varepsilon}}{\longrightarrow} \Sigma_{\varepsilon} \stackrel{\beta_{\varepsilon}}{\longrightarrow} M_{\delta}
$$


is well defined and $\beta_{\varepsilon} \circ \Phi_{\varepsilon}$ is homotopically equivalent to the embedding $\iota: M \rightarrow$ $M_{\delta}$. It follows from Lemmas 3.7 and 3.3, and from Theorem 3.2 that $E_{\varepsilon}$ restricted to $\mathcal{N}_{\varepsilon}$ possesses at least $\operatorname{cat}_{M_{\delta}}(M)$ critical points $u_{i}$. Arguing as in the proof of Corollary 2.5, we conclude that each $u_{i}$ is positive and it is a solution of $\left(\tilde{P}_{\varepsilon}\right)$. The theorem is proved.

\section{Proof of Theorem 1.1}

In order to prove Theorem 1.1 we need to verify that, for $\varepsilon>0$ small enough, the solutions given by Theorem 3.1 satisfy the estimate in (2.3). As in [16], the key step for that is the following.

Proposition 4.1. For any $\varepsilon>0$, let

$$
b_{\varepsilon}^{*}:=\sup \left\{\max _{\partial \Lambda_{\varepsilon}} u_{\varepsilon}: u_{\varepsilon} \in \Sigma_{\varepsilon} \text { is a solution of }\left(\tilde{P}_{\varepsilon}\right)\right\} .
$$

Then $b_{\varepsilon}^{*}$ is finite for $\varepsilon$ small enough and $\lim _{\varepsilon \rightarrow 0^{+}} b_{\varepsilon}^{*}=0$.

Assuming the proposition for a moment, let us see how Theorem 1.1 follows from it.

Proof of Theorem 1.1. Given $\delta>0$ such that $M_{\delta} \subset \Lambda$, we can invoke Theorem 3.1 to obtain, for any $\varepsilon \in\left(0, \varepsilon_{\delta}\right)$ fixed, $\operatorname{cat}_{M_{\delta}}(M)$ solution of $\left(\tilde{P}_{\varepsilon}\right)$. Taking $\varepsilon_{\delta}$ smaller if necessary, we can use Proposition 4.1 to conclude that, if $u_{\varepsilon}$ is one of these solutions, then

$$
u_{\varepsilon}(x)<t_{a} \text { for all } \in \partial \Lambda_{\varepsilon} .
$$

The proof now can be done as in [16]. We recall the argument for completeness. The function $u_{\varepsilon} \in W^{1, p}\left(\mathbb{R}^{N}\right)$ solves the equation

$$
\operatorname{div}\left(a(\varepsilon x)|\nabla u|^{p-2} \nabla u\right)-|u|^{p-2} u+g(\varepsilon x, u)=0 \quad \text { in } \mathbb{R}^{N} .
$$

Let $v_{\varepsilon}$ be defined as $v_{\varepsilon}(x)=\max \left\{u_{\varepsilon}-t_{a}, 0\right\}$ if $x \in \mathbb{R}^{N} \backslash \Lambda_{\varepsilon}, v_{\varepsilon}(x)=0$ otherwise. In view of (4.1), we can take $v_{\varepsilon}$ as a test function in the above equation to get

$$
\int_{\mathbb{R}^{N} \backslash \Lambda_{\varepsilon}} a(\varepsilon x)\left|\nabla v_{\varepsilon}\right|^{p}+c(x) v_{\varepsilon}^{2}+t_{a} c(x) v_{\varepsilon}=0,
$$

where

$$
c(x):=\left|u_{\varepsilon}(x)\right|^{p-2}-\frac{g\left(\varepsilon x, u_{\varepsilon}(x)\right)}{u_{\varepsilon}(x)} .
$$

Condition $\left(g_{3}\right)$ (ii) yields that $c(x) \geq 0$ in $\mathbb{R}^{N} \backslash \Lambda_{\varepsilon}$, hence all the terms in (4.2) are zero. In particular, $v_{\varepsilon} \equiv 0$. Thus, (2.3) holds and $u_{\varepsilon}$ is a solution of $\left(P_{\varepsilon}\right)$. The theorem is proved. 
It remains to prove Proposition 4.1. We commence with a technical convergence result.

Lemma 4.2. Let $\left(\varepsilon_{n}\right) \subset \mathbb{R}^{+}$be such that $\varepsilon_{n} \rightarrow 0$ and $\left(x_{n}\right) \subset \bar{\Lambda}_{\varepsilon_{n}}$. If $u_{\varepsilon_{n}}$ is a solution of $\left(\tilde{P}_{\varepsilon_{n}}\right)$ then, up to a subsequence, $v_{n}:=u_{\varepsilon_{n}}\left(\cdot+x_{n}\right)$ converges uniformly on compact subsets of $\mathbb{R}^{N}$.

Proof. For each $n \in \mathbb{N}$ and $L>0$, we define

$$
\begin{aligned}
v_{L, n} & := \begin{cases}v_{n} & \text { if } v_{n} \leq L, \\
L & \text { if } v_{n} \geq L,\end{cases} \\
z_{L, n} & :=v_{n} v_{L, n}^{p(\beta-1)}, \\
w_{L, n} & :=v_{n} v_{L, n}^{\beta-1},
\end{aligned}
$$

whit $\beta>1$ to be determined later.

Let $a_{n}(x):=a\left(\varepsilon_{n} x+\varepsilon_{n} x_{n}\right)$. Since $u_{\varepsilon_{n}}$ is a solution of $\left(\tilde{P}_{\varepsilon_{n}}\right)$ we have that

$$
\int\left(a_{n}(x)\left|\nabla v_{n}\right|^{p-2} \nabla v_{n} \cdot \nabla \varphi+v_{n}^{p-1} \varphi\right)=\int g\left(\varepsilon_{n} x+\varepsilon_{n} x_{n}, v_{n}\right) \varphi,
$$

for any $\varphi \in X_{\varepsilon}$. Taking $\varphi=z_{L, n}$ we obtain

$$
\begin{aligned}
\int v_{L, n}^{p(\beta-1)} a_{n}\left|\nabla v_{n}\right|^{p}=-p & (\beta-1) \int v_{L, n}^{p(\beta-1)-1} v_{n}\left|\nabla v_{n}\right|^{p-2} \nabla v_{n} \cdot \nabla v_{L, n} \\
& +\int g\left(\varepsilon_{n} x+\varepsilon_{n} x_{n}, v_{n}\right) v_{n} v_{L, n}^{p(\beta-1)}-\int\left|v_{n}\right|^{p} v_{L, n}^{p(\beta-1)} .
\end{aligned}
$$

Since

$$
\int v_{L, n}^{p(\beta-1)-1} v_{n}\left|\nabla v_{n}\right|^{p-2} \nabla v_{n} \cdot \nabla v_{L, n}=\int_{\left\{v_{n} \leq L\right\}} v_{n}^{p(\beta-1)}\left|\nabla v_{n}\right|^{p} \geq 0,
$$

we can use $\left(a_{1}\right)$ to get

$$
a_{0} \int v_{L, n}^{p(\beta-1)}\left|\nabla v_{n}\right|^{p} \leq \int g\left(\varepsilon_{n} x+\varepsilon_{n} x_{n}, v_{n}\right) v_{n} v_{L, n}^{p(\beta-1)}-\int\left|v_{n}\right|^{p} v_{L, n}^{p(\beta-1)} .
$$

Thus, by using (2.4) with $0<\xi<1$, we obtain a constant $C_{1}>0$ such that

$$
\int v_{L, n}^{p(\beta-1)}\left|\nabla v_{n}\right|^{p} \leq C_{1} \int v_{n}^{q} v_{L, n}^{p(\beta-1)} .
$$


On the other hand, by the Sobolev embedding we get

$$
\begin{aligned}
\left|w_{L, n}\right|_{p^{*}}^{p} & \leq C_{2} \int\left|\nabla w_{L, n}\right|^{p}=C_{2} \int\left|\nabla\left(v_{n} v_{L, n}^{\beta-1}\right)\right|^{p} \\
& \leq C_{3}(\beta-1)^{p} \int v_{L, n}^{p(\beta-2)} v_{n}^{p}\left|\nabla v_{L, n}\right|^{p}+C_{3} \int v_{L, n}^{p(\beta-1)}\left|\nabla v_{n}\right|^{p} \\
& =C_{3}(\beta-1)^{p} \int_{\left\{v_{n} \leq L\right\}} v_{L, n}^{p(\beta-1)}\left|\nabla v_{n}\right|^{p}+C_{3} \int v_{L, n}^{p(\beta-1)}\left|\nabla v_{n}\right|^{p} \\
& \leq C_{4} \beta^{p} \int v_{L, n}^{p(\beta-1)}\left|\nabla v_{n}\right|^{p} .
\end{aligned}
$$

This, (4.3), Hölder's inequality and the boundedness of $\left(v_{n}\right)$ imply

$$
\begin{aligned}
\left|w_{L, n}\right|_{p^{*}}^{p} & \leq C_{5} \beta^{p} \int v_{n}^{q} v_{L, n}^{p(\beta-1)}=C_{5} \beta^{p} \int v_{n}^{q-p} w_{L, n}^{p} \\
& \leq C_{5} \beta^{p}\left(\int v_{n}^{p^{*}}\right)^{(q-p) / p^{*}}\left(\int w_{L, n}^{p p^{*} /\left(p^{*}-(q-p)\right)}\right)^{\left(p^{*}-(q-p)\right) / p^{*}} \\
& \leq C_{6} \beta^{p}\left|w_{L, n}\right|_{\alpha^{*}}^{p},
\end{aligned}
$$

with $p<\alpha^{*}=p p^{*} /\left(p^{*}-(q-p)\right)<p^{*}$, whenever $w_{L, n} \in L^{\alpha^{*}}\left(\mathbb{R}^{N}\right)$.

Since $v_{L, n} \leq v_{n}$, we conclude that $w_{L, n} \in L^{\alpha^{*}}\left(\mathbb{R}^{N}\right)$, whenever $v_{n}^{\beta} \in L^{\alpha^{*}}\left(\mathbb{R}^{N}\right)$. If this is the case, it follows from the above inequality that

$$
\begin{aligned}
\left(\int v_{n}^{p^{*}} v_{L, n}^{p^{*}(\beta-1)}\right)^{p / p^{*}} & \leq C_{6} \beta^{p}\left(\int\left(v_{n} v_{L, n}^{\beta-1}\right)^{\alpha^{*}}\right)^{p / \alpha^{*}} \\
& \leq C_{6} \beta^{p}\left|v_{n}\right|_{\beta \alpha^{*} .}^{\beta p} .
\end{aligned}
$$

By Fatou's lemma in the variable $L$, we get

$$
\left|v_{n}\right|_{\beta p^{*}} \leq\left(C_{7}\right)^{1 / \beta} \beta^{1 / \beta}\left|v_{n}\right|_{\beta \alpha^{*}}<\infty,
$$

whenever $v_{n}^{\beta \alpha^{*}} \in L^{1}\left(\mathbb{R}^{N}\right)$.

We now set $\beta:=p^{*} / \alpha^{*}>1$ and note that, since $v_{n} \in L^{p^{*}}\left(\mathbb{R}^{N}\right)$, the above inequalities hold for this choice of $\beta$. Thus, since $\beta^{2} \alpha^{*}=\beta p^{*}$, it follows that (4.4) also holds with $\beta$ replaced by $\beta^{2}$. Hence,

$$
\left|v_{n}\right|_{\beta^{2} p^{*}} \leq\left(C_{7}\right)^{1 / \beta^{2}} \beta^{2 / \beta^{2}}\left|v_{n}\right|_{\beta^{2} \alpha^{*}} \leq\left(C_{7}\right)^{1 / \beta+1 / \beta^{2}} \beta^{1 / \beta+2 / \beta^{2}}\left|v_{n}\right|_{\beta \alpha^{*}} .
$$

By iterating this process and using that $\beta \alpha^{*}=p^{*}$, we obtain

$$
\left|v_{n}\right|_{\beta^{m} p^{*}} \leq C_{7}^{\sum_{i=1}^{m} \beta^{-i}} \beta^{\sum_{i=1}^{m} i \beta^{-i}}\left|v_{n}\right|_{p^{*}} .
$$


Since $\beta>1$ and $\left(v_{n}\right)$ is bounded, we can take the limit as $m \rightarrow \infty$ to get

$$
\left|v_{n}\right|_{\infty} \leq C_{8} .
$$

Let $\Omega \subset \mathbb{R}^{N}$ be a bounded domain and $\xi>0$. The above inequality and (2.4) imply that

$$
\left|v_{n}^{p-1}-g\left(\varepsilon x+\varepsilon_{n} x_{n}, v_{n}\right)\right| \leq(1+\xi) C_{8}^{p-1}+C_{\xi} C_{8}^{q-1} .
$$

Since $u_{\varepsilon_{n}}$ is a solution of $\left(\tilde{P}_{\varepsilon_{n}}\right)$, we can use the above expression and a result of Di Benedetto [17, Theorem 2] to conclude that, for any compact set $K \subset \Omega$, there exists a constant $\bar{C}_{K, \Omega}$, depending only of $C_{8}, N$, $p$, and $\operatorname{dist}(K, \partial \Omega)$, such that

$$
\left|v_{n}\right|_{C^{0, \alpha}(\Omega)} \leq \bar{C}_{K, \Omega},
$$

for some $0<\alpha<1$. It follows from the Schauder embedding theorem [1, Theorem 1.31] that $v_{n}$ possesses a convergent subsequence in $C_{\text {loc }}^{0}\left(\mathbb{R}^{N}\right)$. The lemma is proved.

Proof of Proposition 4.1. Arguing by contradiction, we suppose that there exists $\left(\varepsilon_{n}\right) \subset \mathbb{R}^{+}$such that $\varepsilon_{n} \rightarrow 0$ and $b_{\varepsilon_{n}}=\infty$. Then we can take a sequence $u_{\varepsilon_{n}} \in \Sigma_{\varepsilon_{n}}$ of solutions of $\left(\tilde{P}_{\varepsilon_{n}}\right)$ such that $u_{\varepsilon_{n}}\left(x_{n}\right) \geq b>0$, for some $b>0$ and $\left(x_{n}\right) \in \partial \Lambda_{\varepsilon_{n}}$.

As in the proof of Lemma 3.6, we have that $E_{\varepsilon_{n}}\left(u_{n}\right) \rightarrow m_{0}$. If $v_{n}:=u_{\varepsilon_{n}}(\cdot+$ $\left.x_{n}\right)$, then $v_{n}-v$ weakly in $X$. Since $v_{n}(0)=u_{\varepsilon_{n}}\left(x_{n}\right) \geq b>0$, Lemma 4.2 implies that $v \neq 0$. If $t_{n}>0$ is such that $w_{n}=t_{n} v_{n} \in \mathcal{M}_{0}$, we can argue as in the proof of Lemma 3.5 to conclude that $I_{0}\left(w_{n}\right) \rightarrow m_{0}$. It follows from Lemma 2.4 that $w_{n} \rightarrow w$ in $X$ and $I_{0}(w)=m_{0}$.

Since $\partial \Lambda$ is compact, we may suppose that $\varepsilon_{n} x_{n} \rightarrow \bar{x} \in \partial \Lambda$. In view of $\left(a_{2}\right)$, we have that $a(\bar{x})>a_{0}$. Thus, the same calculations made in (3.7) give a contradiction and we conclude that $b_{\varepsilon}^{*}<\infty$ for $\varepsilon>0$ small enough.

For the second part we argue by contradiction again and suppose that there exists $\varepsilon_{n} \rightarrow 0^{+}$and $b>0$ with $b_{\varepsilon_{n}}^{*} \geq b>0$. For each $n \in \mathbb{N}$ there exists a solution $u_{\varepsilon_{n}} \in \Sigma_{\varepsilon_{n}}$ of the problem $\left(\tilde{P}_{\varepsilon_{n}}\right)$ in such a way that

$$
\frac{b}{2}=b-\frac{b}{2} \leq b_{\varepsilon_{n}}^{*}-\frac{b}{2} \leq \max _{\partial \Lambda_{\varepsilon n}} u_{\varepsilon_{n}} .
$$

Hence we can take a sequence $\left(x_{n}\right) \subset \partial \Lambda_{\varepsilon_{n}}$ such that $u_{\varepsilon_{n}}\left(x_{n}\right) \geq b / 2>0$. The same argument employed in the first part of the proof gets a contradiction. This finishes the proof.

\section{ACKNOWLEDGEMENT}

The second author was supported by post-doctoral grant no. 04/09232-2 from FAPESP/Brazil. 


\section{REFERENCES}

[1] Robert A. Adams, Sobolev Spaces, Academic Press [A subsidiary of Harcourt Brace Jovanovich, Publishers], New York-London, 1975. MR 0450957 (56 \#9247)

[2] Claudianor O. Alves, Existence and multiplicity of solution for a class of quasilinear equations, Adv. Nonlinear Stud. 5 (2005), 73-86. MR 2117622 (2005h:35081)

[3] Angelo Alvino, Guido Trombetti, and P.-L. Lions, On optimization problems with prescribed rearrangements, Nonlinear Anal. 13 (1989), 185-220, http://dx.doi.org/10.1016/0362-546X(89)90043-6. MR 979040 (90c:90236)

[4] Antonio Ambrosetti, Andrea Malchiodi, and Simone SeCCHi, Multiplicity results for some nonlinear Schrödinger equations with potentials, Arch. Ration. Mech. Anal. 159 (2001), 253-271, http://dx.doi.org/10.1007/s002050100152. MR 1857674 (2002m:35044)

[5] Mohamed Salah Baouendi and Charles Goulaouic, Régularité et théorie spectrale pour une classe d'opérateurs elliptiques dégénérés, Arch. Rational Mech. Anal. 34 (1969), 361-379. MR 0249844 (40 \#3085) (French)

[6] Vieri Benci and Giovanna CERAmi, The effect of the domain topology on the number of positive solutions of nonlinear elliptic problems, Arch. Rational Mech. Anal. 114 (1991), 79-93, http://dx.doi.org/10.1007/BF00375686. MR 1088278 (91j:35102)

[7] _ Multiple positive solutions of some elliptic problems via the Morse theory and the domain topology, Calc. Var. Partial Differential Equations 2 (1994), 29-48, http://dx.doi.org/10.1007/BF01234314. MR 1384393 (97b:35063)

[8] Henri Berestycki, Joo-Paulo Dias, Maria J. Esteban, and Mário Figueira, Eigenvalue problems for some nonlinear Wheeler-DeWitt operators, J. Math. Pures Appl. (9) 72 (1993), 493-515. MR 1239101 (94k:35318)

[9] Paolo Caldiroli and Roberta Musina, On the existence of extremal functions for a weighted Sobolev embedding with critical exponent, Calc. Var. Partial Differential Equations 8 (1999), 365-387, http://dx.doi.org/10.1007/s005260050130. MR 1700269 (2000d:35064)

[10] _ On a variational degenerate elliptic problem, NoDEA Nonlinear Differential Equations Appl. 7 (2000), 187-199, http://dx.doi.org/10.1007/s000300050004. MR 1771466 (2001g:35090)

[11] Jan Chabrowski, Degenerate elliptic equation involving a subcritical Sobolev exponent, Portugal. Math. 53 (1996), 167-177. MR 1397453 (97d:35083)

[12] JAN. CHABROWSKI and JIANFU YANG, Existence theorems for elliptic equations involving supercritical Sobolev exponent, Adv. Differential Equations 2 (1997), 231-256. MR 1424769 (97i:35037)

[13] Silvia Cingolani and MoniCa LAZZO, Multiple semiclassical standing waves for a class of nonlinear Schrödinger equations, Topol. Methods Nonlinear Anal. 10 (1997), 1-13. MR 1646619 (2001a:35162)

[14] Multiple positive solutions to nonlinear Schrödinger equations with competing potential functions, J. Differential Equations 160 (2000), 118-138, http://dx.doi.org/10.1006/jdeq.1999.3662. MR 1734531 (2000j:35079)

[15] Robert Dautray and JaCQUes-Louis Lions, Mathematical Analysis and Numerical Methods for Science and Technology. Vol. 1: Physical Origins and Classical Methods, Springer-Verlag, Berlin, 1990, ISBN 3-540-50207-6, 3-540-66097-6, With the collaboration of Philippe Bénilan, 
Michel Cessenat, André Gervat, Alain Kavenoky and Hélène Lanchon. Translated from the French by Ian N. Sneddon. With a preface by Jean Teillac. MR 1036731 (90k:00004)

[16] Manuel del Pino and PATricio L. Felmer, Local mountain passes for semilinear elliptic problems in unbounded domains, Calc. Var. Partial Differential Equations 4 (1996), 121-137, http://dx.doi.org/10.1007/s005260050031. MR 1379196 (97c:35057)

[17] Emmanuele DiBenedetTo, $C^{1+\alpha}$ local regularity of weak solutions of degenerate elliptic equations, Nonlinear Anal. 7 (1983), 827-850, http://dx.doi.org/10.1016/0362-546X(83)90061-5. MR 709038 (85d:35037)

[18] Nassif Ghoussoub, Duality and Perturbation Methods in Critical Point Theory, Cambridge Tracts in Mathematics, vol. 107, Cambridge University Press, Cambridge, 1993, ISBN 0-52144025-4. MR 1251958 (95a:58021)

[19] MONICA LAZZO, Existence and multiplicity results for a class of nonlinear elliptic problems in $\mathbf{R}^{N}$, Discrete Contin. Dyn. Syst. (2003), 526-535. MR 2018155

[20] GONG BAO LI, Some properties of weak solutions of nonlinear scalar field equations, Ann. Acad. Sci. Fenn. Ser. A I Math. 15 (1990), 27-36. MR 1050779 (91d:35043)

[21] PIERRE-LOUIS LIONS, The concentration-compactness principle in the calculus of variations. The locally compact case. II, Ann. Inst. H. Poincaré Anal. Non Linéaire 1 (1984), 223-283. MR 778974 (87e:49035b) (English, with French summary)

[22] JÜRGEN MOSER, A new proof of De Giorgi's theorem concerning the regularity problem for elliptic differential equations, Comm. Pure Appl. Math. 13 (1960), 457-468. MR 0170091 (30 \#332)

[23] M. K. Venkatesha Murthy and Guido Stampacchia, Boundary value problems for some degenerate-elliptic operators, Ann. Mat. Pura Appl. (4) 80 (1968), 1-122. MR 0249828 (40 \#3069) (English, with Italian summary)

[24] Donato Passaseo, Some concentration phenomena in degenerate semilinear elliptic problems, Nonlinear Anal. 24 (1995), 1011-1025, http://dx.doi.org/10.1016/0362-546X(94)00127-4. MR 1321741 (96a:35066)

[25] Alessio POMPONIO and SimONE SECCHI, On a class of singularly perturbed elliptic equations in divergence form: existence and multiplicity results, J. Differential Equations 207 (2004), 229266, http://dx.doi.org/10.1016/j.jde.2004.06.015. MR 2102664 (2005h:35104)

[26] MARCO SQUASSINA, Spike solutions for a class of singularly perturbed quasilinear elliptic equations, Nonlinear Anal. 54 (2003), 1307-1336, http://dx.doi.org/10.1016/S0362-546X(03)00188-3. MR 1995932 (2004h:35079)

[27] NeIL S. TRUdinger, On Harnack type inequalities and their application to quasilinear elliptic equations, Comm. Pure Appl. Math. 20 (1967), 721-747. MR 0226198 (37 \#1788)

[28] Michel Willem, Minimax Theorems, Progress in Nonlinear Differential Equations and their Applications, 24, Birkhäuser Boston Inc., Boston, MA, 1996, ISBN 0-8176-3913-6. MR 1400007 (97h:58037)

Giovany M. Figueiredo:

Departamento de Matemática

Universidade Federal do Pará

66075-110 Belém-PA, Brazil.

E-MAIL: giovany@ufpa.br 
MARCELO F. FURTAdo:

Departamento de Matemática

Universidade de Brasília

70910-900 Brasília-DF, Brazil

E-MAIL: mfurtado@unb.br

KEY WORDS AND PHRASES: Ljusternik-Schnirelmann theory; penalization method; quasilinear problems; positive solutions

2000 Mathematics Subject Classification: 35J20 (35J60)

Received: July 26th, 2005; revised: September 28th, 2005. 